\title{
Family involvement in outpatient care of the premature newborn
}

\author{
Abstract \\ Objective: To determine the families participation in outpatient care of the premature \\ newborn to the Kangaroo Mother Program.
}

Materials and methods: A qualitative descriptive study was carried out, the population study consisted of 10 families belonging to the program, the collection of the information a semi-structured interview and visits the answers obtained through audio recording were categorized and analyzed in the light of the knowledge on the topic.

Results: It was found that families recognize the benefits of the Program, the need for its participation in the ambulatory care.

Conclusion: The Kangaroo Mother Method promotes the maintenance of the health of these, achieving an improvement in the family dynamics; families with the support and counseling Nursing understand and identify the benefits of the program for the newborn and engage in activities in support mother's work; there is active participation of the husband and the mother figures in the care of the newborn, in which each one plays a different role, contributing to the maintenance of the child health.

Keywords: ambulatory care, premature, participation, family, physical disabilities
Volume 3 Issue I - 2017

\section{Concepcion Amador Ahumada,' Concepcion Herrera Gutierrez, ${ }^{2}$ Luz Del Carmen Padilla Franco $^{3}$ \\ 'Master in Nursing, University of Córdoba, Columbia \\ ${ }^{2}$ Specialist in Maternal Child, University of Córdoba, Columbia \\ ${ }^{3}$ Faculty Health Sciences, University of Cordoba, Columbia}

Correspondence: Amador Ahumada, Master in Nursing,

University of Córdoba, Columbia,

Email conceamador@yahoo.es

Received: May 12, 2017 | Published: June 05, 2017

\section{Introduction}

To the World Health Organization, prematurity, state of development that has a child "when is born before 37 completed weeks of gestation"; the international agency reports that one out of every 10 births corresponds to a premature infant, which involves an annual figure of 15 million premature children of these, nearly 1 million children die, and those who survive may have permanent sequelae that affect their development as physical disabilities, neurological or of learning throughout life, which carries a high social and economic cost to families and society, to reduce this mortality is a task that demands particular care as heat as it cools down with ease, as well as support for breastfeeding has difficulty sucking, as well as basic care to prevent infections and breathing difficulties.

In this measure has been endorsed 36 years ago the Kangaroo Mother Method (MMC) that ${ }^{2}$ allows the newly born premature or low birth weight infants, having access and opportunity to these requirements; it consists in a system of care and standardized protocols, based on the skin to skin contact between the child premature or low weight and his mother; it seeks to empower the mother, father or caregivers responsible for the premature infant, giving it the ability to provide comprehensive care to their children, meeting their physical and emotional needs in the critical initial period of their extra uterine lives and raising their potential survival. ${ }^{3}$ In Colombia, the methodology has been implemented as a national program for the intervention of the premature care. ${ }^{4}$

The Kangaroo Mother Program owes its name to the wild scheme of the newborn care marsupial and immature; it was created for the use in humans in 1979 by the neonatologist Rey Sanabria, in Bogotá city (Colombia), in the absence of incubators and needs cost-effective for the maintenance of the Health of Newborns (NB) premature or low birth weight. The MMC allows the establishment and continuity of power supply with breastfeeding (or supplemented if this is not possible for reasons such as maternal HIV, insufficiency in dairy production), as well as improved confidence and capacity of maternal care, supporting the integration of the newly born into the family and reduces the mortality rate between $30 \%$ and $70 \%{ }^{5}$ The intervention $\mathrm{MMC}$ in the institutions of health care is a complement to the neonatal care and health interventions carried out in the premature newly born and/or low birth weight. ${ }^{6}$

The Scientifics' evidence reports that in Ecuador, the research team formed by Sloan and collaborators through a randomized controlled trial showed lower rates of serious illness among infants between the participants of the MMC (5\%) than those identified in the control group $(18 \%))^{7}$ it has also been shown that with the MMC standard are the sequences of the heart rate and ${ }^{8}$ breathing, the oxygenation, glucose $^{9}$ as well as positive effects on sleep patterns and behavior in children has been skin-to-skin contact with their mothers or caregivers; the results report that these physiological conditions tend to be similar and even greater than those observed in newborn infants separated from their mothers.

The protocols indicate that as soon as the baby is feeding properly, maintain a stable body temperature in the MMC and gaining weight, he/she will be able to go home with his/her mother. Of course, babies will still be premature at the time of receiving the high, so it must be ensured that at home they are subject to regular monitoring by a professional, who initially will make daily visits, which will be spacing to weekly or monthly as they evolve the capabilities of the family to take care of the newborn; this professional will assess the home conditions in which they live, mother and child, family support, community as well as the advice of experienced mothers in $\mathrm{MMC}^{10}$ support which is so essential for all mothers, in particular for the beginner women and teenagers, as well as for mothers with newly born very small or very low birth weight. ${ }^{11-13}$ 
According to the MMC is fundamental the family support at home, both during the period of institutional care and home care, because the mother will need this support to meet other household responsibilities due to their daily activities will be focused on the care of the child which can disrupt their routine; the demands of time involve activities such as the extraction of milk according to indications, breastfeeding on demand, give the child the supplementation of iron and folic acid, sensory stimulation, in addition to the follow-up of the basic requirements of cleaning and personal hygiene, washing hands, quiet environment the baby; adaptations such as several stacked pillows on the bed to the rest of the mother, same posture that reduces the risk of apnea for the newborn. ${ }^{14,15}$

Babies must also be monitored at home in search of the presence of signs of risk that require hospital care as excessive increase or decrease of the body temperature of the child, the signs of hypoxemia, irritability, disorders of consciousness, convulsions, diarrhea, vomiting, and have the availability to move both to the health care center of reference.

It is why that this research study, sought to identify the appropriation of knowledge of child care in the program and the type of family involvement in the care of the premature child; to this extent the family as a whole must focus on the goal of providing the premature or low birth weight $\mathrm{RN}$ care necessary for their well-being by engaging in the growth and development of the newborn.

\section{Materials and methods}

\section{Type of study}

The study was classified descriptive, prospective, with a qualitative approach; Because of the aspects related to the objectives of the MMC program and the participation of the family members were valued in the care using qualitative techniques.

\section{Scenario of the study}

The Kangaroo Mother Program of the main Social Enterprise of the State of the Department of Cordoba (Colombia) As well as the domiciliary spaces of kangaroo families.

\section{Population and sample}

The key informants of this study were 10 breastfeeding mothers located inside the families belonging to the Mother Kangaroo Program of the State-owned company for Social Hospital San Jerónimo in the period between January and March 2016, selected by intentional sampling among those who were active in the Program and with hospital discharge of more than one week Table 1.

Table I Mother's conditions

\begin{tabular}{|c|c|c|c|c|c|c|c|c|c|c|}
\hline Condition & $\mathrm{MCl}$ & MC2 & MC3 & MC4 & MC5 & MC6 & MC7 & MC8 & MC9 & MCIO \\
\hline Mother's Age & 18 & 17 & 23 & 35 & 24 & 18 & 16 & 21 & 31 & 19 \\
\hline Membership in health & $S$ & S & C & S & S & $S$ & S & C & S & C \\
\hline Living with the couple & $\mathrm{Si}$ & No & $\mathrm{Si}$ & No & $\mathrm{Si}$ & $\mathrm{Si}$ & $\mathrm{Si}$ & $\mathrm{Si}$ & $\mathrm{Si}$ & $\mathrm{Si}$ \\
\hline Schooling & IP & BSE & BSE & IP & $\mathrm{CP}$ & IP & $\mathrm{CP}$ & UC & $\mathrm{CP}$ & $\mathrm{CP}$ \\
\hline Time of postpartum on weeks & 11 & 7 & 8 & 8 & 10 & 10 & 7 & 13 & 10 & 8 \\
\hline Time to "exit" in weeks & 9 & 6 & 7 & 6 & 8 & 5 & 5 & 11 & 8 & 6 \\
\hline Attends controls & $\mathrm{Si}$ & $\mathrm{Si}$ & $\mathrm{Si}$ & $\mathrm{Si}$ & $\mathrm{Si}$ & $\mathrm{Si}$ & $\mathrm{Si}$ & $\mathrm{Si}$ & $\mathrm{Si}$ & $\mathrm{Si}$ \\
\hline $\begin{array}{l}\text { Number of members in the family, } \\
\text { including the mother }\end{array}$ & 4 & 5 & 2 & 3 & 6 & 4 & 4 & 3 & 2 & 4 \\
\hline \multicolumn{11}{|l|}{ Conventions } \\
\hline S, Subsidized & \multicolumn{4}{|c|}{ IP, incomplete primary } & \multicolumn{6}{|c|}{ BSE, complete secondary education } \\
\hline C, Contributory & \multicolumn{4}{|c|}{$\mathrm{CP}$, complete primary } & \multicolumn{6}{|c|}{ UC, complete University } \\
\hline
\end{tabular}

Source, interview information

Regarding the obstetric characteristics, the postpartum time ranged from 7 to 13 weeks and the time of having the child at home was between 3 and 9 weeks, enough time for the family to adopt a pattern of behaviors in relation to the Care of the premature at home. As it can be seen, the mothers are young women of maximum 35 years, the majority belongs to the subsidized regime which accounts for the poverty condition of their family nucleus; The predominant schooling was up to the primary level. The informants predominated

over nuclear families, a condition that favored the identification of the forms of family participation in the care of the preterm infant.

The newborns had gestational ages between 31 and 36 weeks, were underweight and four of them received combined Mother Kangaroo Program with incubator stays; two children required supplementation in lactation due to inconveniences with maternal milk production and one case of HIV- positive impairment Table 2.

Table 2 Conditions of preterm infants (PI)

\begin{tabular}{|c|c|c|c|c|c|c|c|c|c|c|}
\hline Condition & PI I & PI 2 & PI 3 & PI 4 & PI 5 & PI 6 & PI 7 & PI 8 & PI 9 & PI 10 \\
\hline Gestational age & 36 & 36 & 32 & 33 & 33 & 31 & 34 & 35 & 34 & 34 \\
\hline Birth weight & 2.310 & 2.230 & 2.100 & 2.150 & 2.100 & 1.850 & 2.200 & 2.400 & 2.420 & 2.140 \\
\hline Exclusive Mother Kangaroo & Yes & Yes & Do not & Yes & Yes & Do not & Yes & Do not & Do not & Yes \\
\hline Exclusive breast feeding & Yes & Yes & Yes & Do not & Yes & Do not & yes & Yes & Do not & yes \\
\hline
\end{tabular}

Source, Interview information 


\section{Unit of analysis}

The family participation in care of premature newborns.

\section{Collection of information}

For the collection of the information, a written permission was requested from the management of the ESE Hospital San Jerónimo, once the permission starts the data capture phase was issued, a meeting was held with the health team in charge of the program to present the objectives, scope and methods of the study. The key informants were also duly informed of the same aspects, taking into account the ethical considerations established by Resolution 8430 of 1993 of the Ministry of Health, signed the consent for the interview and the home visit. The information collection was performed in three moments of interaction with the mother reintegrated into the family as follows:

First moment: it was carried out in the facilities of the Mother Kangaroo Program of the institution of the study, in which the researchers were informed about the families active in the program with children in the discharge; who would act as informants. Subsequently telephone calls were made to obtain the availability of the same realization of the home visit and the consents were signed
Second time: a home visit was made to record the type of family participation at home care and family support received by the mother.

Third moment: a meeting was held with the 10 key informants in the Hospital Program's setting of the study scenario, in order to integrate and exchange experiences during the outpatient care process of the premature and the experiences of each participating family. In this session, the interview technique consisted of 5 open, positively worded questions inviting the kangaroo mother to share her experiences about the participation of the family care of the newborn and their perceptions about the objectives of the MMC at this time of the study were recorded and audio testimonies.

\section{Information processing and analysis}

The records were transcribed and to process the qualitative information we proceeded to classify and group the similar semantic expressions contained in the textual expressions, in order to identify the common categories Table 3. From this exercise, the categories "Help and affection" and "Transient substitution of the mother" and "Welfare and recovery" contained in the responses of the mothers were selected.

Table 3 Analysis scheme

\begin{tabular}{lll}
\hline Literal expression & Semantic content & Documented analysis \\
\hline "To recover" & & $\begin{array}{l}\text { Mothers have assumed the role of family caregivers in } \\
\text { favor of the health status of their newborn children }\end{array}$ \\
$\begin{array}{l}\text { "Keep the growth and } \\
\text { development" }\end{array}$ & Recover normal state & \\
\hline
\end{tabular}

Source, self made

\section{Ethical aspects}

The ethical principles of voluntary participation, veracity, beneficence and autonomy of kangaroo families were taken into account for the development of the research. In compliance with what is described in Article 5 of Chapter I of Resolution 8430 of 1993 and what is contained in Law 911 of 2004, which involved respect for the dignity, integrity and protection of their rights, for this purpose, Informed written ESE Hospital reference program, employees working in the same and participating families.

\section{Results}

Identification of the goals of the Kangaroo Mother Program that has been implemented so that the children continue the growth that would correspond to the missing intrauterine period, protected in the mother's chest, in contact with her skin to skin condition that allows her besides being to maintain the temperature, safety, tranquility and feeding.

These benefits of protection, security and tranquility are those that have been prioritized by mothers, which allowed grouping such benefits to generate the category called "help and affection", which can be observed in the following statements:

\section{“(...) and feels good"}

"That you feel calm with the beat of my heart"

\section{“(...), to protect it"}

In the sense of semantic expressions it is interesting to identify as primate or predominate the affective sphere and the maternal-child bond on the other aspects that the mother and the family learned in the MMC such as the kangaroo position, the time the child should remain in this position, such as clothing, hygiene, medication and the importance of breastfeeding.

Also, mothers who are kangaroos, properly assume the importance of the education they have received from the team of Nursing professionals, incorporating in their knowledge that these actions of direct care are impact on the well-being and recovery of the condition of their premature newborn; The mothers express their knowledge and they feel in expressions that denote the understanding of their role as family caregivers.

Family Care with the content of the expressions, which denote the task of the family members in care of the preterm infant, it was generally appreciated that also father and grandmothers participate in the newborn care.

The actions care performed by the grandmothers of the child are limited to "caring for the child" for short periods while the mother carries out her basic daily activities such as feeding, hygiene, dressing and so on, which has been categorized as "transient substitution of the mother".

In the same sense of caring actions, it is identified that the father is the only member of the family who interacts directly with his child, who dedicates time and shares his energy in the process of maintaining his/her body heat through direct contact; In addition, it provides the mother with a support system expressed as an accompaniment to attendance to the program controls, taking into account the statements made by:

"My husband loves it and he goes with me to the controls" 
“(...) my mother-in-law helps me with the child when I need to do my things."

\section{"My husband takes care of the child at night"}

"My husband does not go with me to the controls because he works."

In support actions, a negative influence of the educational level of the father, which affects the socioeconomic conditions, alters the type of accompaniment performed by the father of the premature child. In Colombia, by jurisprudence the father has the right to a time of leave to support the woman in the first days of the perinatal; Nevertheless it is observed in these cases the parents are forced to work, in order to guarantee the family sustenance; Due to this condition husbands often meet the woman and their children at night hours, this allows the mother a breath and rest and simultaneously with the assumption of her responsibilities, the father is linked to his newly born child, born through a series of emotive actions. In both cases in which the father figure is absent, the home care of the premature newborn is exclusively provided by women in the family, being the mother and the maternal grandmother who usually stay closer and aware of the needs of her daughter and grandchild.

As for the other members of the family - different from the father and grandmothers -, it was possible to identify that they are kept at a prudent distance from the newborn, although they are frequently "observed", they are attentive to its evolution but do not contact it directly; this apparent isolation from the rest of the family is a form of preventive and intentional care that in no way expresses isolation from the child, but rather expresses an attempt by the family to "protect him/her from infection"; It could be observed that the participation that the rest of the family provides to the kangaroo mother is done through support actions, also in substitution of the mother, but in the sense of performing the domestic actions that should be performed by the woman who participates in the MMC.

\section{Discussion}

The results largely support Klauss and Kennel's "Good Attachment" hypothesis about the psycho-affective advantage provided to the neonate; fostering the symbiotic relationship between the subject and his caretaker. ${ }^{16}$

The behavior of each family members of those children, in the type of support that they received by the mother coincides with the importance Pava, attaches to the social structure of kinship in the child's survival, demonstrated in that they are relatives in the first degree of consanguinity who directly involve themselves as caregivers in support of the mother, in this case the father and the maternal and/ or paternal grandmothers in whom the mother can delegate direct functions of care to the newborn; While the other family members perform activities that allow the mother-caregiver the availability of key time spaces for a truce in the baby care. ${ }^{17}$

The rest of the family with their practice of apparent isolation of the newborn expresses protective practices in response to the experience of vulnerability of the premature baby that they perceive of the members of the family; The findings indicate that nursing support is required for the extended family to carry out appropriate activities that allow the inclusion of the new member in the family nucleus; explaining them the precautions and measures required to safeguard the integrity of the newborn, while at the same time integrating him/ her into family life since the discharge of the newborn; in this sense a family with sufficient support and counseling can affectively link the newborn and actively participate in care improving family dynamics.

It is evident that the affection that family feels towards him, motivates the family and it is translated in the acceptance of the new responsibilities and commitments that implies the participation in the Kangaroo Mother Program, the role that each member of the household fulfills in ambulatory care of the preterm newborn is for the mother according to what Castiblanco said, in being a Kangaroo involves a series of direct actions like massaging, protecting the cold, feeding the child, maintaining hygiene, preserving the protection of the environment, giving love, providing supplemental oxygen to the baby if required and know about the child's signs; however, it has been observed in the present study that there are external factors that disrupt the dynamics of care such as the absence of the father figure when the parents are working, the woman is a single mother or do not have the family support. ${ }^{18}$ The active participation of the family in the care of prematurity at an ambulatory level, as proposed by Romo, highlights the role of the mother in actions aimed at providing warmth, love and exclusive breastfeeding. ${ }^{19}$

\section{Conclusion}

The process of adapting the family for the outpatient care of the newborn within the Kangaroo Mother Program requires education and counseling to encourage the participation of the whole family and adjusting the family and personal-social dynamics to overcome gestational immaturity and achieve Having a full term child in the preterm delivery is a pleasant experience.

Families understand and identify the benefits provided by the Newborn and Self-Care Program, evidenced by the acceptance of the educational support and counseling recommendations they receive from nursing and the interdisciplinary team leading the program, subjects involved in the direct care of premature or low birth weight newborns have been able to acquire basic knowledge that allows them to carry out the activities correctly.

The active participation of the family in outpatient care of the newborn is concentrated in father, mother, grandmothers; the uncles, cousins and other relatives participate indirectly releasing the mother of some of her household responsibilities.

\section{Acknowledgements}

None.

\section{Conflict of interest}

The author declares no conflict of interest.

\section{References}

1. World health organization. What is a premature child? Geneva, Switzerland: World health organization; 2014.

2. Conde Agudelo A, Belizán JM, Diaz-Rosello J. Kangaroo mother care to reduce morbidity and mortality in low birthweight infants. Cochrane Database Syst Rev. 2011:(3):CD002771.

3. World Health Organization. Mother kangaroo method. WHO document. Geneva, Switzerland: World Health Organization; 2014.

4. Charpak N, Ruíz JG, Ángel MI, et al. Lineamientos técnicos para la implementación de Programas Madre Canguro en Colombia. Ministerio de la Protección Social de Colombia - Programa Mundial de Alimentos; 2009. 
5. Rey ES, Martinez HG. Manejo racional del niño prematuro. En: Universidad Nacional, Curso de Medicina Fetal. Colombia: Bogotá, Universidad Nacional; 1983.

6. McInnes RJ, J Chambers. Infants admitted to neonatal units-interventions to improve breastfeeding outcomes: a systematic review 19902007. Maternal and Child Nutritio. 2008;4(4):235-263.

7. Sloan NL, E Pinto Rojas, C Stern, et al. Kangaroo mother method: randomised controlled trial of an alternative method of care for stabilised low-birthweight infants. The Lancet. 1994;344(8925):782-785.

8. De Leeuw R, Colin EM, Dunnebier EA, et al. Physiologic effects of kangaroo care in very small preterm infants. Biol Neonate. 1991;59(3):149155

9. Ludington Hoe SM, Hadeed AJ, Anderson GC. Physiologic responses to skin-to-skin contact in hospitalized premature infants. $J$ Perinatol. 1991;11(1):19-24.

10. Organización Mundial de la Salud. Método Madre Canguro. Guía Práctica. Ginebra.

11. Wahlberg V, Affonso D, Persson B. A retrospective, comparative study using the kangaroo method as a complement to the standard incubator care. European Journal of Public Health. 1992;2(1):34-37.

12. Fischer C, Sontheimer D, Scheffer F, et al. Cardiorespiratory stability of premature boys and girls during kangaroo care. Early Hum Dev. 1998;52(2):145-153
13. Acolet D, Sleath $\mathrm{K}$, Whitelaw A. Oxygenation, heart rate and temperature in very low birth weight infants during skin-to-skin contact with their mothers. Acta Paediatr Scand. 1989;78(2):189-193.

14. http://apps.who.int/iris/bitstream/10665/43083/1/9243590359.pdfPág.

15. Jenni OG, Von Siebenthal K, Wolf M, et al. Effect of nursing in the head elevated tilt position (15 degrees) on the incidence of bradycardic and hypoxemic episodes in preterm infants. Pediatrics. 1997;100(4):622-625.

16. Klaus, M y Kennel, J Pais/bebê. A formação do apego (D. Batista, Trad). Porto Alegre: Artes Médicas. En: Psicologia: Reflexão e Crítica. 2002;15(3):625-635.

17. Pava Laguna C. Prácticas de cuidado con el recién nacido prematuro o bajo peso, que ofrecen las madres en el hogar. Tesis de investigación presentada como requisito para optar al título de: Magister en Enfermería; 2013.

18. Castiblanco López N y Muñoz de Rodríguez L. Visión de las madres en el cuidado del hijo prematuro en el hogar. En: Avances en Enfermería. 2011;29(1):120-129.

19. Romo FF, Valdés AIM, Rodríguez LAR. Implementación del método "Madre Canguro" como alternativa a la atención convencional en neonatos de bajo peso. Implementation of "Kangaroo-Mother" care method as an alternative to conventional care infants with low birth weight. Mediciego. 2012;18(1):1. 